\title{
Research on Ordering and Transportation of Raw Materials in Production Enterprises Based on Goal Programming
}

\author{
Jiankang $\mathrm{LI}^{\mathrm{a}}$,Yufei CHEN ${ }^{\mathrm{b}}$, Kexuan $\mathrm{LI}^{\mathrm{a}}$ and Yangzhuo CHEN ${ }^{\mathrm{a}, 1}$ \\ a School of Automation and Electronic Information, Xiangtan University, China \\ ${ }^{\mathrm{b}}$ School of Mathematics and Computational Sciences, Xiangtan University, China
}

\begin{abstract}
In the current complex economic situation, production enterprises are facing manyuncertainties. Reasonable planning of the ordering and transportation of raw materials to ensure the stable operation of the production supply chain is the key to the sustainable development of enterprises. In this paper, a TOPSIS model based on entropy weight and a raw material ordering and transportation model for production enterprises based on multi-objective programming are proposed to provide reference for raw material ordering and transportation selection of production enterprises. In this model, the supply characteristics of suppliers are quantitatively analyzed, and on the basis of the demand of production enterprises, the supplier is determined based on the principle of minimum number of suppliers. Then, the future transshipment loss rate of the transporter is predicted based on the time series, and then the corresponding transporter and transshipment scheme are determined with the lowest loss rate as the target. The research on the model of suppliers and transporter has a good guiding significance for the ordering and transportation of raw materials in manufacturing enterprises.
\end{abstract}

Keywords. Multiobjective programming, TOPSIS, Supplier characteristic analysis.

\section{Introduction}

With the arrival of economic globalization, raw material suppliers are becoming more and more important to the development of production enterprises. the reasonable management of raw material ordering is the key to ensure the quality of raw materials and stable supply of goods, which is more conducive to improve the profits of enterprises. Therefore, through reasonable planning for the selection of suppliers and transporters of raw materials, the formulation of appropriate ordering and transportation strategy is an important factor for the sustainable development of production enterprises.

Supplier selection is a process of comprehensive evaluation and selection of suppliers by production enterprises. the main methods to evaluate suppliers are fuzzy evaluation model, grey comprehensive evaluation, Analytic hierarchy process (AHP), etc., but these methods are tedious and subjective. In order to select raw material suppliers scientifically and objectively, this paper adjusts the subjective deviation by entropy method, quantitatively determines a series of index weights and optimal values of raw material suppliers, and then uses TOPSIS to approximate the ideal solution to

${ }^{1}$ Corresponding Author, Yangzhuo CHEN, School of Automation and Electronic Information, Xiangtan University, China ;E-mail:271896208@qq.com. 
study them, so as to realize the selection of suppliers. On this basis, a single objective programming model is established with the lowest loss rate as the goal to get the corresponding transfer plan.

\section{Ordering and Transportation Problem Description}

The raw materials used by a building and decorative plate manufacturing enterprise can be divided into three categories A, B and C. it is necessary to make a 24-week raw material ordering and transfer plan in advance. determine the raw material supplier and the corresponding weekly raw material order quantity according to its production capacity and entrust the transporter to transfer to the enterprise warehouse.

For manufacturing enterprises, the production capacity is 28200 cubic meters per week, and each cubic meter of products needs to consume the original output of Class A, or Class B raw materials, or Class $\mathrm{C}$ raw materials. The purchase unit price of category $\mathrm{A}$ and $\mathrm{B}$ raw materials is $20 \%$ and $10 \%$ higher than that of Class $\mathrm{C}$, respectively, and the unit cost of transportation and storage of the three types of raw materials is the same;For suppliers, due to the particularity of materials, it is not guaranteed to supply goods strictly according to the order quantity, so the enterprise should purchase all the raw materials actually provided by the supplier;For transporters, the transshipment capacity of each transporter is 6000 cubic meters per week, and there is a certain loss of raw materials in the actual transshipment process. The ordering and transportation of raw materials of the manufacturer are shown in figure 1.

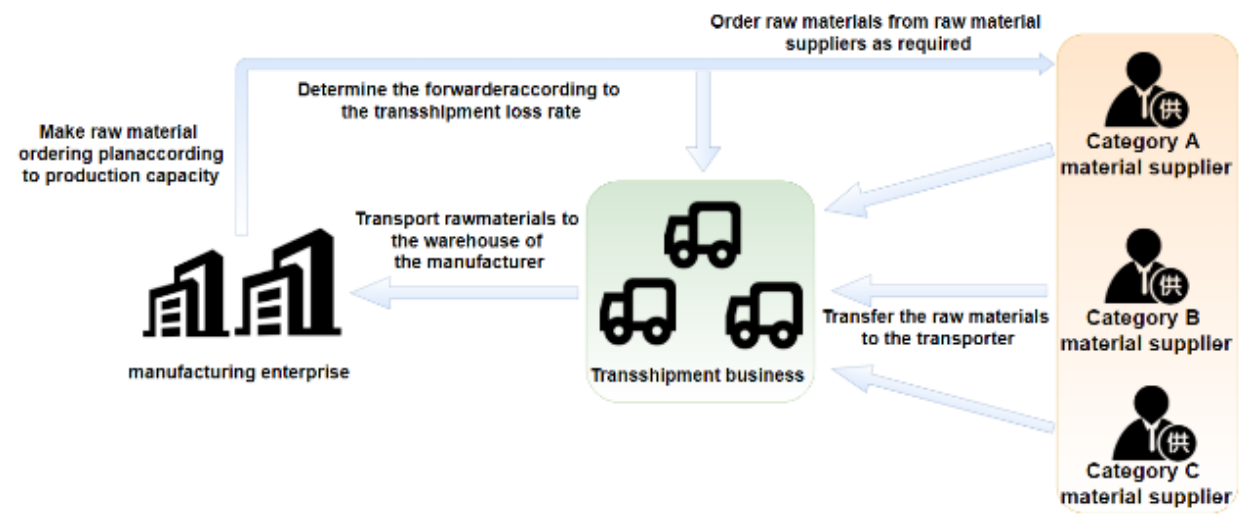

Figure 1. Ordering and transportation process. 


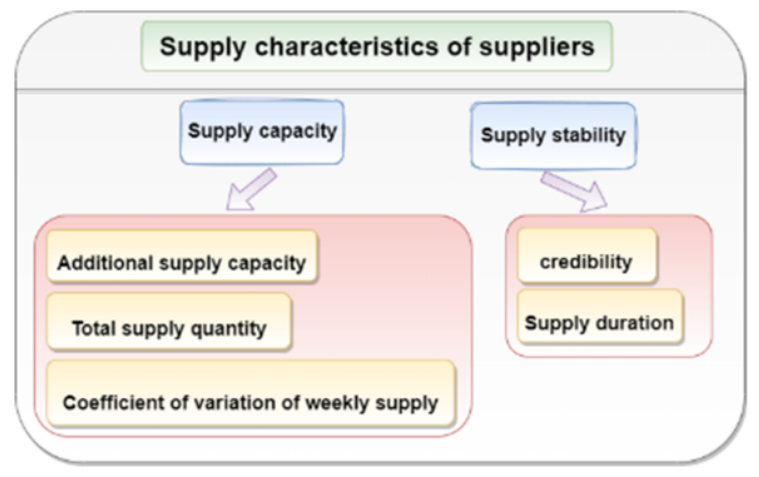

Figure 2. Supply characteristics of suppliers

\section{Analysis of Supply Characteristics of Suppliers}

\subsection{Unify the Supply Capacity of Different Raw Materials}

In order to analyze the capacity satisfaction of category A, B and C raw materials, we unify them to the completion degree of the production capacity of the production enterprise according to the corresponding material consumption per cubic meter, then the energy supply of category A, B and C raw materials is treated as follows:

$$
Y_{A i}=\frac{x_{A i}}{0.6} \quad Y_{B i}=\frac{x_{B i}}{0.66} \quad Y_{C i}=\frac{x_{C i}}{0.72}
$$

Where: $x_{A i}, x_{B i}, x_{C i}$ represent the raw material supply quantity of the $i \sim t h$ supplier of Class A, B and $\mathrm{C}$ respectively; $Y_{A i}, Y_{B i}, Y_{C i}$ represent the unified production energy of the raw material supply of the $i \sim t h$ supplier of class $\mathrm{A}, \mathrm{B}$ and $\mathrm{C}$ respectively.

\subsection{Supplier Characteristic Variable Selection}

For the supplier's supply characteristics, mainly from the supply capacity and supply stability to analyze the aracteristics of the supplier [1-2], as shown in figure 2 above.

\subsubsection{Coefficient of Variation of Weekly Supply}

The coefficient of variation of weekly supply quantity refers to the coefficient of variation of suppliers' weekly supply capacity (quantity of raw materials) calculated on the basis of weeks. it is used to measure the degree of change of suppliers' supply quantity. The formula is as follows:

$$
\beta_{i}=\frac{\sigma_{i}}{\mu_{i}}
$$

Where: $\beta_{i}$ represent the coefficient of variation of the weekly supply quantity of the $i \sim t h$ supplier; $\mu_{i}$ is the average weekly supply. 


\subsubsection{Additional Supply Capacity}

Additional supply capacity refers to the average weekly supply of suppliers in the past five years in addition to the order volume of additional supply, used to represent the supplier's potential supply capacity in the last five years. The formula is as follows:

$$
\delta_{i}=\frac{\sum_{i=1}^{n}\left(y_{i}-x_{i}\right)}{n_{i}}
$$

Where: $\delta_{i}$ represents $i \sim t h$ supplier's total supply capacity; $x_{i}$ is the quantity of raw materials supplied by the supplier in $i \sim t h$ week; $y_{i}$ is the raw material order quantity of the enterprise $i \sim t h$ week; $n$ is the total number of weeks supplied by suppliers in recent five years.

\subsubsection{Total Supply Quantity}

Total supply refers to the total production capacity of A, B and C raw material suppliers to enterprise products in the past five years. After the unified standard, the total supply of all kinds of raw material suppliers can reflect their supply capacity.

\subsubsection{Credibility}

The credibility of suppliers is an important index to measure the supply capacity of suppliers, the higher the credibility of suppliers, the more trustworthy of enterprises. The formula is as follows:

$$
R_{i}=\frac{m_{i}}{n_{i}}
$$

Where: $m_{i}$ represents the number of weeks of supply completed by the $i \sim t h$ supplier as required; $R_{i}$ represents the credibility of the $i \sim t h$ supplier.

\subsubsection{Supply Duration}

Supply duration refers to the total number of weeks of cooperation between the supplier and the enterprise in the past five years, which is helpful to reflect the enterprise's recognition of the supplier. The longer the cooperation duration, the higher the enterprise's recognition of the supplier. The formula is as follows:

$$
\begin{gathered}
\varepsilon_{i}= \begin{cases}1, & x_{i}>0 \\
0, & x_{i}<0\end{cases} \\
T_{i}=\sum_{i=1}^{n} \varepsilon_{i}
\end{gathered}
$$


Where: $\varepsilon_{i}$ represents the supply situation of the first supplier: "1" means that there is supply for the enterprise in that week, " 0 " means that there is no supply for the enterprise in that week ; $T_{i}$ represents the total number of weeks supplied by the $i \sim t h$ supplier in the recent five years.

\subsection{Quantitative Analysis of Supplier's Supply Characteristics}

Before the quantitative analysis of supply characteristics, it is necessary to determine the weight of each index. in order to avoid the adverse influence of human subjective factors on the quantitative analysis of supply characteristics[1], this paper uses Entropy weight method(EWM) to determine the weights of each supply characteristic index, and then according to the obtained weights, TOPSIS is used to quantify the supply characteristics of each supplier.

\subsubsection{Calculation of Supply Feature Weight by Ewm}

a. Data normalization and normalization

$$
\alpha_{i j}=\frac{x_{i j}-x_{\min }}{x_{\max }-x_{\min }}
$$

b. Calculate the entropy value of the index

$$
p_{i j}=\frac{\alpha_{i j}}{\sum_{i=1}^{n} \alpha_{i j}} \quad 0 \leqslant p_{i j} \leqslant 1
$$

c. Calculate the entropy value and difference degree of the $\mathrm{j}$-th index

$$
\begin{gathered}
e_{j}=-\frac{1}{\ln n} \sum_{i=1}^{n} p_{i j} \ln \left(p_{i j}\right) \\
d_{j}=1-e_{j}
\end{gathered}
$$

d. Calculate entropy weight

$$
W_{j}=\frac{d_{j}}{\sum_{j=1}^{n} d_{j}}
$$

The weights of the five characteristic indicators of the supplier are calculated, as shown in the following table1:

Table 1. The weights of the characteristic indicators of the supplier

\begin{tabular}{cccccc}
\hline Index & $Y_{i}$ & $\beta_{i}$ & $\delta_{i}$ & $R_{i}$ & $T_{i}$ \\
\hline Weight & 0.701089 & 0.058949 & 0.000764 & 0.08556 & 0.153638 \\
\hline
\end{tabular}




\subsubsection{Quantifying Supply Characteristics with Topsis}

a. Construct weighting matrix

$$
\alpha_{i j}^{*}=\alpha_{i j} \cdot w_{j}
$$

b. Find the best and worst solution

Find out the maximum value of each column of characteristic index, the component vector, representing the ideal supplier;At the same time, find out the minimum value of each column of characteristic index, the component vector, representing the least ideal supplie.

$$
\left\{\begin{array}{l}
\alpha_{i j}^{*_{+}}=\max _{n, p}\left(z_{1}^{*_{+}}, z_{2}^{*_{+}}, \cdots, z_{m}^{*_{+}}\right) \\
\alpha_{i j}^{*_{-}}=\min _{n, p}\left(z_{1}^{*_{-}}, z_{2}^{*_{-}}, \cdots, z_{m}^{*_{-}}\right)
\end{array}\right.
$$

c. Optimal and worst distance

Define the distance between the i-th supplier and the ideal target as $D_{i}^{+}$, and the distance between the non-ideal target as $D_{i}^{-}$.

$$
\left\{\begin{array}{l}
D_{i}^{*+}=\sqrt{\sum_{j=1}\left(z_{i j}^{*}-z_{j}^{*^{+}}\right)^{2}} \\
D_{i}^{*_{-}}=\sqrt{\sum_{j=1}\left(z_{i j}^{*}-z_{j}^{*_{-}}\right)^{2}}
\end{array}\right.
$$

d. Calculate the score

Define the score of the $i \sim t h$ supplier, which is calculated as follows:

$$
C_{i}=\frac{D_{i}^{+}}{D_{i}^{+}-D_{i}^{-}} \quad 0 \leqslant C_{i} \leqslant 1
$$

The closer the $C_{i}$ is to 1 , the stronger the supplier's supply capability.

\section{Ordering Based on The Principle of Economic Optimization and Minimum Loss}

We select 28 suppliers, determine the raw material ordering plan according to the economic optimal principle, then determine the transshipment scheme with the least loss according to the material supply ordered with the minimum loss principle as the goal.

\subsection{Determine the Ordering Scheme}

According to the analysis of the ratio of all kinds of raw materials consumed per unit product and the unit price ratio, when considering the ordering of raw materials with the best economy, the priority is $\mathrm{A}=\mathrm{C}>\mathrm{B}$. 
a. Determine decision variables

In order to ensure that the supplier can meet the production of the enterprise, we make decisions on the average weekly supply capacity $\bar{x}_{i}$ and completion rate of the supplier $\psi_{i j}$. The specific calculation formula is:

$$
\begin{aligned}
& \bar{x}_{i}=\frac{\delta_{i}}{n_{i}} \\
& \psi_{i j}=\frac{x_{i}}{y_{i}} \times 100 \%
\end{aligned}
$$

Where: $\delta_{i}$ represents the total supply volume of the $i \sim t h$ supplier; $n_{i}$ Rrepresents the total supply weeks of the $i \sim t h$ supplier; $\psi_{i j}$ represents the completion rate of the $i \sim t h$ supplier in the $j \sim t h$ week.

b. Determine the objective function

$$
\min z=\sum_{1 \leqslant i \leqslant 28,1 \leqslant j \leqslant 24} Y_{i j} \cdot \psi_{i j} \cdot b_{i}
$$

Where: $Y_{i j}$ represents the order quantity of raw materials of the $i \sim t h$ enterprise in the $j \sim t h$ week; $b_{i}$ represents the unit price of materials supplied by the $i \sim t h$ supplier.

c. Determine constraints

Considering the production capacity of the enterprise and the actual supply capacity of the supplier, the goal programming model based on the optimal economy is obtained:

$$
\begin{aligned}
& \min z=\sum_{1 \leqslant i \leqslant 28,1 \leqslant j \leqslant 24} Y_{i j} \cdot \psi_{i j} \cdot b_{i} \\
& \text { s.t. }\left\{\begin{array}{l}
\sum_{1 \leqslant i \leqslant 28} Y_{i j} \geqslant 28837 \quad(j=1,2, \cdots, 24) \\
Y_{i j} \leqslant \bar{x}_{i}
\end{array}\right.
\end{aligned}
$$

The supplier with the lowest weekly material ordering cost is obtained by using MATLAB, and the raw material ordering scheme based on the principle of economic optimization is obtained. 


\subsection{Transfer Scheme Based on Minimum Loss Rate}

Because of the uncertainty of the transporter's loss rate, we need to predict the transshipment loss rate of each transporter in the next 24 weeks based on the transshipment data in the previous five years.

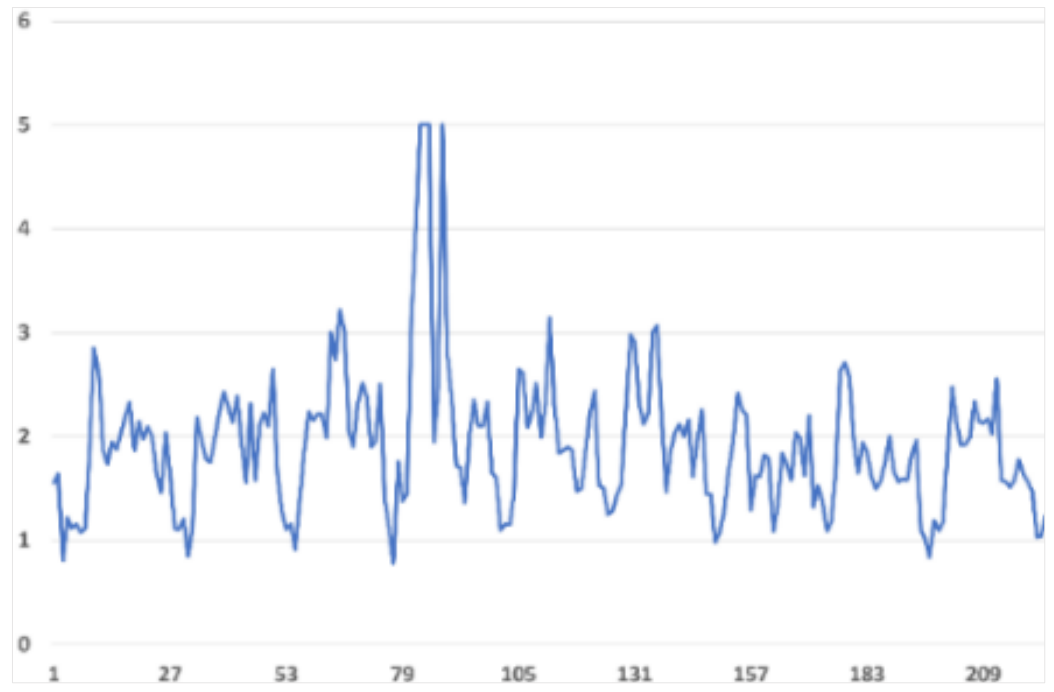

Figure 3. The loss rate data of 240 weeks

The loss rate data of 240 weeks of transporters are statistically analyzed, and the scatter diagram is obtained, from which it can be seen that there is a certain law in the loss rate of each transporter. Excluding the influence of extreme loss rate, the stability trend of loss rate can be obtained.

As can be seen from figure 3, the whole transshipment process is cyclical. There are 10 cycles in the last five years, and each cycle is 24 weeks. Therefore, the transshipment loss rate of the transshipment company $k$ in the $j \sim t h$ week can be expressed by $\bar{L}(k, j)$ (the mean turnover loss rate of the $j \sim t h$ week in the previous 10 cycles), and the calculation formula is as follows:

$$
L_{k}=\bar{L}(k, j)
$$

According to the loss rate of each transporter, in order to realize the scheme of minimizing transshipment loss, the selection of transporter and its specific week arrangement are planned and analyzed.

a. Determine decision variables

In order to optimize the supply capacity, we make a decision analysis on $m_{i j}$ (the transshipment volume of the transporters).

b. Determine the objective function

$$
\min z=\sum_{1 \leqslant i \leqslant 8,1 \leqslant j \leqslant 24} m_{i j} \gamma_{i j}
$$


Where: $m_{i j}$ represents the transshipment volume of the $i \sim t h$ transporter in $j \sim t h$ first week; $\gamma_{i j}$ represents the loss rate of the first transporter in the first week.

\section{c. Determine constraints}

Considering the actual transshipment capacity of the forwarder, the following constraints are obtained:

$$
\begin{aligned}
& m_{i j} \leqslant 6000 \quad(i=1,2 、 \cdots, 8 ; j=1,2 、 \cdots, 24) \\
& \text { s.t. } \quad m_{i j} \leqslant 6000 \quad(i=1,2 、 \cdots, 8 ; j=1,2 、 \cdots, 24)
\end{aligned}
$$

Finally, the transporter and its volume in the next 24 weeks are obtained by using MATLAB.

\section{Conclusion}

There is still room for improvement and optimization in the work of this paper, It can be seen that supplier selection and raw material transfer scheme are complex modeling problems. It involves many factors, especially when encountering emergencies such as COVID-19 and the state power restriction policy, the uncertainties and unknown factors of suppliers will increase significantly.

\section{References}

[1] Lo, H. W., Liaw, C. F., Gul, M., \& Lin, K. Y. (2021). Sustainable supplier evaluation and transportation planning in multi-level supply chain networks using multi-attribute-and multi-objective decision making. Computers \& Industrial Engineering, 107756.

[2] Sivanagaraju Pitchaiah Dusanapudi,Hussaian Manzoor \& Govardhan D.(2020).A review on multi attribute decision making for evaluation and selection of supplier for materials. Materials Today: Proceedings(prepublish), doi:10.1016/J.MATPR.2020.07.201. 